\title{
PREVALÊNCIA E RESISTÊNCIA A ANTIBIÓTICOS DE (Streptococcus equi) DA CA VIDADE NASAL DE EQUINOS HÍGIDOS NO MUNICÍPIO DE FERNANDOPÓLIS, SÃO PAULO, BRASIL
}

\author{
[Prevalence and antibiotic resistance of (Streptococcus equi) from nasal cavity of healthy equines]
}

\author{
Augusto Marsola Pansani ${ }^{1}$, Igor Renan Honorato Gatto ${ }^{2}$, Danila Fernanda Rodrigues Frias ${ }^{3 *}$, Dora Inés \\ Kozusny-Andreani ${ }^{3}$ \\ ${ }^{1}$ Médico Veterinário autônomo. \\ ${ }^{2}$ Departamento de Medicina Veterinária Preventiva, Universidade Estadual Paulista, Jaboticabal, SP, Brasil. \\ ${ }^{3}$ Universidade Camilo Castelo Branco (UNICASTELO), Fernandópolis, SP, Brasil.
}

\begin{abstract}
RESUMO - A adenite equina, popularmente chamada de garrotilho, é uma enfermidade contagiosa, causada pela bactéria Streptococcus equi. A enfermidade é conhecida por ter alta morbidade e baixa letalidade e seus prejuízos econômicos devem-se à perda de performance e alto custo do tratamento. O objetivo deste trabalho foi avaliar a presença e o perfil de resistência a antibióticos de Streptococcus equi da cavidade nasal de equinos hígidos da região de Fernandópolis - SP. Foram utilizados 50 equinos hígidos sem raça definida e com idade entre 12 a 24 meses. De cada animal foram colhidas amostras de exsudato da cavidade nasal por meio de swabs esterilizados e em seguida colocados em tubos contendo ágar base acrescido com 5\% de sangue ovino desfibrinado. Os isolados que apresentaram colônias mucosas e $\beta$-hemolíticas foram analisados pela coloração de Gram, submetidos ao teste da catalase, e identificados pelo sistema API Rapid ID 32 Strep. Todos os isolados foram avaliados em difusão em placa. Foram isolados sessenta e sete amostras de Streptococcus equi, das subespécies equi e zooepidemicus. Verificou-se prevalência maior de $S$. equi subesp zooepidemicus (p < 0,001). A maioria dos isolados de $S$. equi subespécies equi foram mutirresistentes, havendo diferenças significativas entre os diferentes antibióticos ( $\mathrm{p}<0,005)$, enquanto que foi verificada maior susceptibilidade nos isolados $S$. equi subespécies zooepidemicus ( $\mathrm{p}<0,05$ ). Os resultados obtidos mostraram que ambas as subespécies de Streptococcus equi fazem parte da microbiota normal da cavidade nasal de equinos, e que as mesmas diferem quanto a susceptibilidade aos antibióticos.
\end{abstract}

Palavras-Chave: Adenite equina; equinos; Streptococcus equi; susceptibilidade.

\begin{abstract}
Strangles is a disease of the respiratory tract of horses caused by Streptococcus equi. The disease has high morbidity and low lethality, and produces economic losses due to low performance and high cost treatment. This research focused on evaluate antibiotic resistance of Streptococcus equi from the nasal cavity of healthy horses from the region Fernandópolis - São Paulo. Fifty (50) mongrel healthy horses, ageing from twelve to twenty four (12-24) months were used. Samples from the nasal cavity of each animal were obtained using sterile swabs and after were cultured on blood agar base plus 5\% defibrinated sheep blood. Plates that showed mucous colonies and $\beta$ - hemolytic were tested using Gram stain method, the catalase test, and identified by the system API Rapid ID thirty-two 32 Strep. All isolates were analyzed by plate difusion. Sixty seven of the obtained isolates were Streptococcus equi subspecies equi and zooepidemicus. There was higher prevalence of $S$. subsp equi zooepidemicus ( $\mathrm{p}<0.001$ ). Most isolates of $S$. equi subsp equi were antibiotical multiresistant with significant differences between different antibiotics $(\mathrm{p}<0.005)$, whereas higher susceptibility was observed in isolated S. subsp equi zooepidemicus (p < 0.05). The results showed that both subspecies of Streptococcus equi are part of the normal flora of the nasal cavity of horses, and that they differ in susceptibility to antibiotics.
\end{abstract}

Keywords: Strangles; horses; Streptococcus equi; susceptibility.

\footnotetext{
* Autor para correspondência. E-mail: danilafrias@ yahoo.com.br Recebido: 04 de março de 2016.

Aceito para publicação: 19 de abril de 2016.
} 


\section{INTRODUÇÃO}

O gênero Streptococcus é um grupo de bactérias Gram-positivas, catalase negativos, anaeróbios facultativos e imóveis, que formam cadeias de diferentes comprimentos, e podem infectar muitas espécies animais, causando infecções supurativas como mastite, adenite, metrite, poliartite e meningite (Hirsh \& Zee, 2003; Tortora et al., 2012).

Streptococcus equi subespécie equi, agente etiológico da adenite, também conhecida como garrotilho, em equinos é uma bactéria $\beta$-hemolítica pertencente ao grupo $\mathrm{C}$ de Lancefield, podendo ser diferenciado de $S$. equisimilis e de $S$. equi subsp zooepidemicus pela fermentação de trealose, sorbitol, lactose e maltose (Kuwamoto et al., 2001, Timoney, 2004). Pesquisas recentes sobre homologia de DNA demonstraram que $S$. equi subesp equi evoluiu do ancestral de $S$. equi subesp zooepidemicus, ambas as espécies bacterianas estão fenotipicamemte e genotipicamente relacionadas (Facklam, 2002, Webb et al., 2008, Quinn et al., 2011, Waller et al., 2011).

A bactéria $S$. equi subesp equi afeta somente equídeos (Waller et al., 2011), enquanto que $S$. equi subesp. zooepidemicus é considerado um microorganismo comensal oportunista do trato respiratório superior, capaz de causar doenças secundárias, como problemas respiratórios e pneumonias em equinos e em outras espécies, incluindo o homem (Holden et al., 2009, Rasmussen et al., 2013, Fulde \& Valentin-Weigand, 2013).

A adenite equina é uma doença altamente contagiosa entre os equídeos e apresenta alta morbidade, baixa letalidade, podendo ocasionar perdas econômicas importantes principalmente pelo alto custo do tratamento (Moloney et al., 2013). A doença caracteriza-se pela formação de abscessos nos linfonodos regionais do trato respiratório superior que podem eventualmente romper, supurando pela pele, ou drenando o conteúdo pelo trato respiratório superior (Harrington et al., 2002, Waller \& Jolley 2007, Waller et al., 2011; Quinn et al., 2011). Além dos casos típicos de adenite, foram relatados casos de encefalites em equinos causadas por infecções disseminadas de S. equi subesp. Equi, além de hemiplegia laringeana, broncopneumonia, púrpura hemorrágica, timanimso das bolsas guturais (Goehring et al., 2005). Este agente infeccioso possui fatores de virulência que auxiliam na ocorrência da enfermidade, são eles: cápsula de ácido hialurônico, estreptolisinas, hialuronidase, proteína M antifagocítica e estreptoquinases (Harrington et al., 2002, Quinn et al., 2011).
É importante salientar, que o S. equi subesp. equi é um agente zoonótico para humanos que são eventualmente infectados através do contato com equinos portadores ou doentes (Timoney, 2004). Esse agente é frequentemente isolado do trato respiratório superior de equinos saudáveis (Rasmussen et al., 2013).

O diagnóstico da doença é realizado por meio da avaliação dos sinais clínicos e pela demonstração do agente em esfregaços de exsudato nasal ou pus. A confirmação do diagnóstico é realizada com o isolamento da bactéria a partir do material proveniente das lesões ou órgãos afetados (Schild, 2001).

Após o diagnóstico da doença, é recomendada terapêutica com antimicrobianos. Sabe-se que o $S$. equi subsp equi é sensível a penicilina G, ceftiofur, cloranfenicol, eritromicina, lincomicina e às tetraciclinas (Quinn et al., 2011), no entanto o surgimento de cepas resistentes é inevitável. Assim, o objetivo deste trabalho foi isolar Streptococcus equi da cavidade nasal de equinos hígidos da região de Fernandópolis - SP, e caracterizar seu perfil de susceptibilidade a antibióticos.

\section{MATERIAL E METODOS}

A pesquisa foi desenvolvida no Laboratório de Microbiologia da Unicastelo entre agosto de 2014 e julho de 2015. O projeto foi encaminhado a Comissão de Ética para Uso de Animais CEUA/Unicastelo e o mesmo foi aprovado sob $\mathrm{n}^{\circ}$ $1-00009 / 2012$.

Foram utilizados 50 equinos hígidos avaliados por exame clínico direto, provenientes de 15 propriedades da região de Fernandópolis, SP, sem raça definida e com idade entre 12 a 24 meses. De cada animal foram colhidas amostras de exsudatos da cavidade nasal por meio de swabs esterilizados e material obtido foi acondicionado em meio Stuart para o transporte. Para esta finalidade os animais foram contidos em brete e com auxílio de swabs foram colhidas amostras em duplicata, friccionando suavemente a mucosa nasal evitando ocasionar desconforto e dor para o animal. As amostras foram acondicionadas em caixa isotérmica contendo gelo reutilizável para transporte do local da colheita ao laboratório, onde o processamento das mesmas foi imediato.

Cada amostra foi cultivada separadamente em meio base de ágar sangue (BAS, Oxoid®) acrescido de $5 \%$ de sangue ovino desfibrinado e incubado a $37^{\circ} \mathrm{C}$ por 48 horas, em condições aeróbicas e anaeróbicas. Os isolados que apresentaram colônias mucosas e $\beta$-hemolíticas foram analisados pela coloração de Gram em microscópio de luz, os cocos 
Gram-positivos agrupados em cadeia foram submetidos ao teste da catalase utilizando-se peróxido de hidrogênio (3\%). As colônias mucosas, beta-hemolíticas, catalase negativa, contendo cocos Gram positivos foram repicadas no meio de cultura BAS, incubadas a $37^{\circ} \mathrm{C}$ por 24 horas para classificação por meio da cor, densidade, superfície, hemólise e tamanho das colônias de acordo com a metodologia proposta por Koneman et al. (2001).

As colônias morfologicamente caracterizadas como $S$. equi, sendo que a densidade bacteriana inicial foi determinada pela absorbância a 550nm usando a escala de McFarland standard (BioMérieux, Marcyl'Etiole, France) que correspondeu aproximadamente a $1,5 \times 10^{8} \mathrm{UFC} \mathrm{mL}^{-1}$. A partir desta solução foram realizadas diluições seriadas resultando em concentração de $1,5 \times 10^{6} \mathrm{UFC} \mathrm{mL}^{-1}$, e esta suspensão empregada para inoculação da placa do sistema API Rapid ID 32 Strep, (BioMérieux, Marcy-l'Etiole, France) de acordo com as instruções do fabricante, e os resultados obtidos após 4 a 24 horas de incubação a $37^{\circ} \mathrm{C}$. Cada isolado foi também semeado em Phenol Red Broth Base (DIFCO, Michigan, USA) contendo 1 $\%$ de trealose, sorbitol e lactose, incubados a $37^{\circ} \mathrm{C}$ por 48 horas quando foi determinada a fermentação dos carboidratos. Amostras da cepa padrão de Streptococcus equi subesp. equi ATCC 39506, foram empregadas como referência.

O método de Kirby Bauer Modificado foi utilizado para verificação da susceptibilidade antimicrobiana in vitro (BAUER \& KIRBY, 1966), sendo avaliados os antimicrobianos amicacina $(30 \mu \mathrm{g})$, estreptomicina $(10 \mu \mathrm{g}), \quad$ gentamicina $(10 \mu \mathrm{g})$; amoxicilina $(10 \mu \mathrm{g})$, ampicilina $(10 \mu \mathrm{g})$, cefalexina $(30 \mu \mathrm{g})$, cefalotina $(30 \mu \mathrm{g})$, imipenem $(10 \mu \mathrm{g})$, oxacilina $(1 \mu \mathrm{g})$, penicilina (10 UI); enrofloxacina $(5 \mu \mathrm{g})$; sulfazotrim $(25 \mu \mathrm{g})$ e tetraciclina $(30 \mu \mathrm{g})$. Os resultados foram interpretados de acordo com parâmetros pré-estabelecidos (NCCLS, 2003).

De acordo com a metodologia descrita por Krumperman (1983), foi calculado o índice de resistência múltipla aos antimicrobianos (IRMA). O IRMA foi calculado pela razão entre o número de antibióticos aos quais o isolado foi resistente e o número de antibióticos ao qual o isolado foi exposto. Quando o IRMA é superior a 0,2 caracteriza multirresistência.

Os dados obtidos foram tabulados e submetidos a analise da variância e a comparação das médias pelo teste de Duncan a $5 \%$ de probabilidade, utilizando o software ASSISTAT, desenvolvido pela Assistat Assistência Estatística (Silva \& Azevedo, 2009).

\section{RESULTADOS E DISCUSSÃO}

Das amostras colhidas da cavidade nasal dos 50 equinos utilizados foram obtidos, após cultivo, 67 isolados. As colônias na sua maioria mucoides e translucidas apresentaram diâmetros que variaram entre 1,0 a 4,0mm. Quanto à coloração, 27 isolados apresentaram cor amarela e 40 a cor branca. Colônias mucoides de $S$. equi são virulentas, a subespécie zooepidemicus são tipicamente não mucoides, porém isolados frescos podem apresentar certa mucosidade (Sweeney et al., 2005).

Por meio do sistema API Rapid ID 32 Strep e pela fermentação dos carboidratos trealose, sorbitol e lactose, os isolados com colônias de cor amarela foram identificados como pertencentes à espécie Streptococcus equi subesp. equi, não fermentadores de trealose, lactose e sorbitol, os de colônias brancas e fermentadores de lactose e sorbitol como S. equi subesp. zooepidemicus. Não foi constatada variação no padrão fermentativo dos carboidratos nos isolados de $S$. equi. Estes resultados são contrários aos obtidos por Kirinus et al. (2011) que verificaram que de 38 isolados, dois de Streptococcus equi subesp. equi fermentaram a lactose. Isso pode indicar que a mesma subespécie da bactéria demonstra diferentes padrões fermentativos de carboidratos.

Do total de 67 isolados (Tabela 1), 14,9\% continham unicamente $S$. equi subesp.equi, 34,3\% S. equi subesp. zooepidemicus e 50,8\% continham ambas as subespécies, verificando-se prevalência de $S$. equi subesp zooepidemicus ( $<<0,001)$.

Tabela 1. Espécies de Streptococcus equi isolados da cavidade nasal de equinos hígidos.

\begin{tabular}{lcc}
\hline Espécies bacterianas & Número de isolados & \% de isolados \\
\hline Streptococcus equi subesp equi & $10 \mathrm{c}$ & 14,9 \\
S. equi subesp zooepidemicus & $23 \mathrm{a}$ & 34,3 \\
S.equi subesp equi+S. zooepidemicus & $17 \mathrm{~b}$ & 50,8 \\
\hline Total & 67 & 100 \\
\hline \multicolumn{2}{c}{ C.V.\% } &
\end{tabular}

*Letras iguais na mesma coluna não diferem estatisticamente pelo teste de Duncan com 5\% de probabilidade.

Estes resultados foram condicentes com os obtidos Mir et al. (2013) que ao avaliarem a microbiota da cavidade nasal de equinos observaram que $17,44 \%$ dos isolados corresponderam a $S$. equi subesp zooepidemicus e $1,24 \%$ a $S$. equi subesp equi. Resultados semelhantes foram obtidos por 
Jannatabadi et al. (2008) e Christley et al. (2010) que também verificaram predominância desta subespécie em amostras da cavidade nasal de equinos com doenças respiratórias. Manzoor et al. (2008) verificaram que de 70 amostras obtidas em seu estudo, com amostras coletadas de nódulos e abcessos de cavalos, 54\% apresentaram S. equi subesp.equi, $11 \%$ S. equi subesp zooepidemicus, e em $11 \%$ foram isoladas $S$. equi subesp.equi e $S$. equi subesp.equsimilis. Esses dados demonstram que a bactéria $S$. equi subesp. equi, e a $S$. equi subesp. zooepidemicus estão presentes na cavidade nasal tanto de equinos com doenças respiratórias como também de animais hígidos, podendo ser transmitidas aos seres humanos, o que o torna um importante agente zoonótico para a saúde pública.
A avaliação da susceptibilidade das duas subespécies de Streptococcus equi (Tabelas 2 e 3), mostrou que houve menor resistência aos antibióticos da subespécie zooepidemicus quando comparada com a subespécie equi ( $<<0,05)$, sendo que os índices de resistência múltipla aos antimicrobianos (IRMA) foram de $42,5 \%$, e de $70 \%$ respectivamente. Estes resultados foram semelhantes aos obtidos por Kirinus et al. (2011) que verificaram maior índice de resistência em $S$. equi subespécie equi. Isso pode estar relacionado ao fato da adenite equina ser causada pelo $S$. equi subespécie equi, e o tratamento instituído ser baseado no uso empírico e indiscriminado de antibióticos.

Tabela 2. Susceptibilidade e índice de resistência múltipla a diferentes classes de antimicrobianos de Streptococcus equi subespécie equi. isolados da cavidade nasal de equinos hígidos.

\begin{tabular}{|c|c|c|c|c|c|c|c|c|c|c|c|c|c|c|c|c|c|c|}
\hline \multirow{4}{*}{ Amostras } & \multicolumn{18}{|c|}{ ANTIMICROBIANOS } \\
\hline & $\mathrm{A}$ & $\mathrm{E}$ & G & $\mathrm{A}$ & $\mathrm{A}$ & $\mathrm{C}$ & $\mathrm{C}$ & I & $\mathrm{O}$ & $\mathrm{P}$ & $\mathrm{E}$ & $\mathrm{L}$ & $\mathrm{E}$ & $\mathrm{P}$ & $\mathrm{R}$ & $\mathrm{S}$ & $\mathrm{T}$ & \\
\hline & M & S & $\mathrm{E}$ & M & M & $\mathrm{E}$ & $\mathrm{E}$ & M & $\mathrm{X}$ & $\mathrm{E}$ & $\mathrm{N}$ & I & $\mathrm{R}$ & $\mathrm{O}$ & I & $\mathrm{U}$ & $\mathrm{E}$ & IRMA \\
\hline & $\mathrm{I}$ & $\mathrm{T}$ & $\mathrm{N}$ & $\mathrm{O}$ & $\mathrm{P}$ & $\mathrm{F}$ & $\mathrm{L}$ & $\mathrm{I}$ & A & $\mathrm{N}$ & $\mathrm{R}$ & $\mathrm{N}$ & I & $\mathrm{L}$ & $\mathrm{F}$ & $\mathrm{T}$ & $\mathrm{T}$ & \\
\hline $1,5,15,23$ & $\mathrm{~S}$ & $\mathrm{~S}$ & S & $\mathrm{R}$ & $\mathrm{R}$ & $\mathrm{S}$ & $\mathrm{S}$ & $\mathrm{S}$ & $\mathrm{S}$ & $S$ & $\mathrm{~S}$ & $\mathrm{I}$ & I & $\mathrm{S}$ & $\mathrm{S}$ & $\mathrm{S}$ & $\mathrm{R}$ & 0,18 \\
\hline $2,3,4,24$ & $\mathrm{~S}$ & $\mathrm{R}$ & $S$ & $\mathrm{R}$ & $\mathrm{R}$ & $\mathrm{S}$ & $\mathrm{S}$ & $\mathrm{R}$ & $\mathrm{S}$ & $S$ & $\mathrm{~S}$ & $\mathrm{~S}$ & S & $\mathrm{S}$ & I & $\mathrm{S}$ & $\mathrm{R}$ & 0,29 \\
\hline $6,8,9$ & $\mathrm{~S}$ & $\mathrm{~S}$ & S & $\mathrm{S}$ & $\mathrm{S}$ & $\mathrm{R}$ & $\mathrm{S}$ & I & S & $\mathrm{R}$ & $\mathrm{S}$ & $\mathrm{S}$ & $\mathrm{S}$ & I & I & $S$ & $\mathrm{R}$ & 0,18 \\
\hline 7,25 & $\mathrm{R}$ & $\mathrm{S}$ & S & $\mathrm{S}$ & $\mathrm{R}$ & $\mathrm{S}$ & $\mathrm{S}$ & $\mathrm{R}$ & S & $\mathrm{R}$ & $\mathrm{S}$ & $\mathrm{S}$ & $\mathrm{S}$ & $\mathrm{S}$ & $S$ & $S$ & $\mathrm{~S}$ & 0,24 \\
\hline 10,14 & $\mathrm{~S}$ & $\mathrm{~S}$ & S & $\mathrm{S}$ & $\mathrm{S}$ & $\mathrm{R}$ & $\mathrm{S}$ & $\mathrm{I}$ & $\mathrm{R}$ & I & $\mathrm{S}$ & $\mathrm{S}$ & $\mathrm{S}$ & $\mathrm{R}$ & $\mathrm{R}$ & $\mathrm{R}$ & $\mathrm{R}$ & 0,35 \\
\hline $11,16,22$ & $\mathrm{R}$ & $\mathrm{R}$ & S & $\mathrm{R}$ & $\mathrm{R}$ & $\mathrm{R}$ & $\mathrm{S}$ & $\mathrm{S}$ & $\mathrm{S}$ & $\mathrm{S}$ & $\mathrm{S}$ & $\mathrm{R}$ & $\mathrm{S}$ & $\mathrm{S}$ & $\mathrm{R}$ & $\mathrm{S}$ & $\mathrm{S}$ & 0,41 \\
\hline $12,13,17$ & $\mathrm{~S}$ & $\mathrm{~S}$ & S & $\mathrm{S}$ & $\mathrm{R}$ & $\mathrm{S}$ & $\mathrm{R}$ & $\mathrm{R}$ & S & $S$ & I & I & $\mathrm{S}$ & $\mathrm{S}$ & $\mathrm{S}$ & $\mathrm{S}$ & $\mathrm{R}$ & 0,24 \\
\hline $18,19,26$ & $\mathrm{R}$ & $\mathrm{R}$ & S & $\mathrm{S}$ & $\mathrm{S}$ & $\mathrm{R}$ & S & $\mathrm{S}$ & I & $S$ & S & $\mathrm{R}$ & $\mathrm{S}$ & $\mathrm{S}$ & $\mathrm{S}$ & $\mathrm{S}$ & $\mathrm{S}$ & 0,29 \\
\hline 20 & $\mathrm{~S}$ & $\mathrm{R}$ & $\mathrm{R}$ & I & I & $\mathrm{S}$ & $S$ & $\mathrm{R}$ & S & $S$ & I & $\mathrm{S}$ & $\mathrm{R}$ & $\mathrm{R}$ & $\mathrm{S}$ & $\mathrm{S}$ & S & 0,29 \\
\hline 21,27 & I & $\mathrm{R}$ & $S$ & $\mathrm{R}$ & $\mathrm{R}$ & $\mathrm{R}$ & $S$ & $\mathrm{~S}$ & $\mathrm{~S}$ & $\mathrm{~S}$ & $\mathrm{~S}$ & I & I & $\mathrm{S}$ & $\mathrm{S}$ & $\mathrm{S}$ & $\mathrm{R}$ & 0,29 \\
\hline ATCC* & $\mathrm{R}$ & $\mathrm{R}$ & $\mathrm{R}$ & $\mathrm{S}$ & $\mathrm{S}$ & $\mathrm{S}$ & $S$ & $\mathrm{~S}$ & $\mathrm{~S}$ & $\mathrm{~S}$ & $\mathrm{R}$ & $\mathrm{S}$ & $\mathrm{S}$ & $\mathrm{S}$ & $\mathrm{S}$ & $\mathrm{S}$ & $\mathrm{S}$ & 0,24 \\
\hline
\end{tabular}

*Cepa padrão Streptococcus equi subesp equi ATCC 39506. AMI = amicacina, EST = estreptomicina, GEN = gentamicina, $\mathrm{AMO}=$ amoxicilina, $\mathrm{AMP}=$ ampicilina, $\mathrm{CEF}=$ cefalexina, $\mathrm{CEL}=$ cefalotina, $\mathrm{IMP}=$ imipenem, OXA = oxacilina, PEN = penicilina, ENR = enrofloxacina, LIN = lincosamida, ERI = eritromicina, $\mathrm{POL}=$ polimixina $\mathrm{B}, \mathrm{RIF}=$ rifampicina, $\mathrm{SUT}=$ sulfazotrim, $\mathrm{TET}=$ tetraciclina. $\mathrm{IRMA}=$ índice de resistência múltipla a antimicrobianos.

No presente estudo os isolados apresentaram perfil de resistência variável, com diferenças significativas entre os mesmos, $(\mathrm{p}>0,05)$. Entre os diferentes antibióticos avaliados verificou-se que $100 \%$ (27) dos isolados de S. equi subsp equi foram susceptíveis a enrofloxacina, enquanto que $66,7 \%$ $(18 / 27)$ e $63 \%$ (17/27) foram resistentes a ampicilina e tetraciclina respectivamente, (Tabela 2). Estes resultados corroboram os obtidos por Manzoor et al. (2008) que demostraram resistência a tetraciclina em $100 \%$ dos isolados, não entanto divergem dos observados por Sweeney et al. (2005), que encontraram sensibilidade a ampicilina em $100 \%$ (191) dos isolados de S. equi subsp equi. Maior susceptibilidade foi observada para os antibióticos gentamicina, eritromicina (26/27), oxacilina, sulfazotrim (25/27), cefalotina, polimixina B (24/27), penicilina, rifampicina $(22 / 27)$ e lincosamida (21/27). Os resultados obtidos nesta pesquisa diferem dos estudos realizados por Sweeney et al. (2005) nos Estados Unidos de América, Manzoor et al. (2008) no Paquistão e Kirinus et al. (2011) no Brasil onde estes autores verificaram susceptibilidade a penicilina em todos os isolados. Em relação a oxacilina verificou-se que existem resultados divergentes, os isolados estudados por Sweeney et al. (2005) foram $100 \%$ susceptíveis, enquanto que os avaliados por Kirinus et al. (2011) houve resistência a este antibiótico em $8 \%$ dos isolados, enquanto no presente estudo $7,4 \%$ dos isolados apresentaram resistência. A variabilidade no padrão de resistência e susceptibilidade a antibióticos pela bactéria em estudo pode estar relacionada a origem da amostra avaliada. No local de estudo, comumente usa-se tetraciclina para tratamentos de diversas enfermidades. Como a bactéria foi isolada de animais hígidos, que nunca apresentaram a 
doença, sendo assim, nunca foram tratados, a mesma entra em contato rotineiramente com o fármaco citado devido a outras doenças infecciosas, desenvolvendo assim, resistência.

Tabela 3. Susceptibilidade e índice de resistência múltipla a diferentes classes de antimicrobianos de Streptococcus equi subespécie zooepidemicu isolados da cavidade nasal de equinos hígidos.

\begin{tabular}{|c|c|c|c|c|c|c|c|c|c|c|c|c|c|c|c|c|c|c|}
\hline \multirow{4}{*}{ Amostras } & \multicolumn{18}{|c|}{ ANTIMICROBIANOS } \\
\hline & $\mathrm{A}$ & $\mathrm{E}$ & $\mathrm{G}$ & $\mathrm{A}$ & $\mathrm{A}$ & $\mathrm{C}$ & $\mathrm{C}$ & $\mathrm{I}$ & $\mathrm{O}$ & $\mathrm{P}$ & $\mathrm{E}$ & $\mathrm{L}$ & $\mathrm{E}$ & $\mathrm{P}$ & $\mathrm{R}$ & $\mathrm{S}$ & $\mathrm{T}$ & \\
\hline & M & $\mathrm{S}$ & $\mathrm{E}$ & M & M & $\mathrm{E}$ & $\mathrm{E}$ & M & $\mathrm{X}$ & $\mathrm{E}$ & $\mathrm{N}$ & I & $\mathrm{R}$ & $\mathrm{O}$ & I & $\mathrm{U}$ & $\mathrm{E}$ & IRMA \\
\hline & $\mathrm{I}$ & $\mathrm{T}$ & $\mathrm{N}$ & $\mathrm{O}$ & $\mathrm{P}$ & $\mathrm{F}$ & $\mathrm{L}$ & $\mathrm{P}$ & A & $\mathrm{N}$ & $\mathrm{R}$ & $\mathrm{N}$ & $\mathrm{I}$ & $\mathrm{L}$ & $\mathrm{F}$ & $\mathrm{T}$ & $\mathrm{T}$ & \\
\hline $1,2,4,15$ & $\mathrm{~S}$ & $S$ & $\mathrm{~S}$ & $\mathrm{~S}$ & I & $S$ & S & $\mathrm{S}$ & $\mathrm{S}$ & $\mathrm{I}$ & $\mathrm{S}$ & $\mathrm{S}$ & $\mathrm{S}$ & $\mathrm{S}$ & $S$ & $\mathrm{~S}$ & S & 0,00 \\
\hline $3,6,33,40$ & $\mathrm{~S}$ & $\mathrm{~S}$ & $\mathrm{~S}$ & $\mathrm{~S}$ & $\mathrm{~S}$ & $\mathrm{R}$ & S & I & $\mathrm{S}$ & $\mathrm{S}$ & $\mathrm{S}$ & $\mathrm{R}$ & $\mathrm{S}$ & $\mathrm{S}$ & $\mathrm{S}$ & $\mathrm{S}$ & $\mathrm{S}$ & 0,12 \\
\hline $5,7,10,16$ & $\mathrm{~S}$ & $\mathrm{R}$ & $\mathrm{S}$ & $\mathrm{S}$ & $\mathrm{S}$ & $\mathrm{S}$ & I & $\mathrm{S}$ & I & I & $\mathrm{S}$ & $\mathrm{S}$ & $\mathrm{S}$ & $\mathrm{S}$ & $\mathrm{S}$ & $\mathrm{S}$ & $\mathrm{S}$ & 0,06 \\
\hline $8,23,36$ & $\mathrm{R}$ & $\mathrm{S}$ & $\mathrm{S}$ & $\mathrm{S}$ & $\mathrm{R}$ & $\mathrm{R}$ & I & I & $\mathrm{S}$ & $\mathrm{S}$ & $\mathrm{S}$ & $\mathrm{R}$ & $\mathrm{R}$ & $\mathrm{S}$ & $\mathrm{S}$ & $\mathrm{S}$ & $\mathrm{S}$ & 0,29 \\
\hline $9,20,24$ & $\mathrm{~S}$ & $\mathrm{~S}$ & $\mathrm{~S}$ & $\mathrm{~S}$ & $\mathrm{R}$ & $\mathrm{S}$ & S & $\mathrm{S}$ & $\mathrm{S}$ & $\mathrm{S}$ & $S$ & $\mathrm{R}$ & $\mathrm{R}$ & $\mathrm{R}$ & $\mathrm{S}$ & $\mathrm{S}$ & $\mathrm{S}$ & 0,23 \\
\hline $11,35,37$, & $\mathrm{S}$ & $\mathrm{R}$ & $\mathrm{S}$ & $\mathrm{S}$ & $\mathrm{R}$ & $\mathrm{R}$ & $\mathrm{S}$ & $\mathrm{R}$ & $\mathrm{R}$ & $\mathrm{S}$ & $\mathrm{S}$ & I & I & $\mathrm{S}$ & I & $\mathrm{S}$ & $\mathrm{S}$ & 0,29 \\
\hline $12,13,14$ & $\mathrm{~S}$ & $\mathrm{R}$ & $\mathrm{S}$ & $\mathrm{S}$ & $\mathrm{R}$ & $\mathrm{S}$ & S & I & I & $\mathrm{S}$ & $\mathrm{S}$ & $\mathrm{R}$ & I & I & $\mathrm{S}$ & $\mathrm{R}$ & $\mathrm{S}$ & 0,23 \\
\hline $17,19,20$ & $\mathrm{R}$ & $\mathrm{S}$ & $\mathrm{S}$ & $\mathrm{S}$ & $\mathrm{S}$ & $\mathrm{S}$ & S & $\mathrm{S}$ & S & $\mathrm{S}$ & $\mathrm{S}$ & $\mathrm{S}$ & $\mathrm{S}$ & $\mathrm{S}$ & S & $\mathrm{S}$ & $\mathrm{S}$ & 0,06 \\
\hline $18,25,26,27$ & $\mathrm{~S}$ & $\mathrm{R}$ & $\mathrm{S}$ & $\mathrm{R}$ & $\mathrm{R}$ & $\mathrm{R}$ & S & $\mathrm{S}$ & $\mathrm{R}$ & $S$ & $\mathrm{R}$ & $\mathrm{S}$ & $\mathrm{S}$ & I & S & $\mathrm{S}$ & $\mathrm{R}$ & 0,41 \\
\hline $21,38,39$ & $\mathrm{~S}$ & $\mathrm{~S}$ & $\mathrm{~S}$ & $\mathrm{~S}$ & $\mathrm{~S}$ & I & S & $\mathrm{S}$ & $\mathrm{S}$ & I & I & $\mathrm{R}$ & $\mathrm{S}$ & $\mathrm{S}$ & S & $\mathrm{S}$ & I & 0,06 \\
\hline 28 & $\mathrm{~S}$ & $\mathrm{R}$ & $\mathrm{R}$ & $\mathrm{R}$ & $\mathrm{S}$ & $\mathrm{S}$ & I & $\mathrm{R}$ & I & $\mathrm{S}$ & $\mathrm{S}$ & $\mathrm{R}$ & $\mathrm{R}$ & $\mathrm{S}$ & $\mathrm{R}$ & $\mathrm{S}$ & I & 0,41 \\
\hline 29,34 & $\mathrm{~S}$ & $\mathrm{~S}$ & $\mathrm{~S}$ & $\mathrm{~S}$ & I & $\mathrm{S}$ & I & I & $\mathrm{S}$ & $\mathrm{S}$ & $\mathrm{R}$ & I & $\mathrm{S}$ & $\mathrm{S}$ & S & I & $\mathrm{S}$ & 0,06 \\
\hline 30 & $\mathrm{~S}$ & I & $\mathrm{S}$ & S & $\mathrm{S}$ & $\mathrm{S}$ & S & $\mathrm{S}$ & I & $\mathrm{S}$ & $\mathrm{S}$ & I & $\mathrm{S}$ & $\mathrm{S}$ & I & $\mathrm{S}$ & $\mathrm{S}$ & 0,00 \\
\hline 31,32 & $\mathrm{~S}$ & $\mathrm{~S}$ & $\mathrm{~S}$ & S & $\mathrm{S}$ & $S$ & I & $\mathrm{R}$ & I & $S$ & $\mathrm{~S}$ & $\mathrm{~S}$ & $\mathrm{~S}$ & $\mathrm{~S}$ & S & $\mathrm{S}$ & $\mathrm{S}$ & 0,06 \\
\hline ATCC* & $\mathrm{R}$ & $\mathrm{R}$ & $\mathrm{R}$ & $\mathrm{S}$ & $\mathrm{S}$ & $S$ & $\mathrm{~S}$ & $S$ & $\mathrm{~S}$ & $S$ & $\mathrm{R}$ & $\mathrm{S}$ & $\mathrm{S}$ & $\mathrm{S}$ & $\mathrm{S}$ & $\mathrm{S}$ & $\mathrm{S}$ & 0,24 \\
\hline
\end{tabular}

*Cepa padrão Streptococcus equi subesp zooepidemicus ATCC 39506. AMI = amicacina, EST = estreptomicina, GEN = gentamicina, $\mathrm{AMO}=$ amoxicilina, $\mathrm{AMP}=$ ampicilina, $\mathrm{CEF}=$ cefalexina, $\mathrm{CEL}=$ cefalotina, $\mathrm{IMP}=$ imipenem, OXA = oxacilina, PEN = penicilina, ENR = enrofloxacina, LIN = lincosamida, ERI = eritromicina, $\mathrm{POL}=$ polimixina $\mathrm{B}, \mathrm{RIF}=$ rifampicina, $\mathrm{SUT}=$ sulfazotrim, $\mathrm{TET}=$ tetraciclina. $\mathrm{IRMA}=$ índice de resistência múltipla a antimicrobianos.

O perfil de susceptibilidade de Streptococcus equi subespécie zooepidemicus (Tabela 3) foi superior ao de $S$. equi subespécie equi (Tabela 2), IRMA superior a 0,20 foi verificado em $42,5 \%$ e $74,1 \%$ dos isolados, respectivamente, diferindo significativamente entre ambas as subespécies ( $\mathrm{p}<$ $0,05)$.

Todos os isolados de Streptococcus equi subespécie zooepidemicus apresentaram susceptibilidade a penicilina (Tabela 3), corroborando os resultados obtidos por Manzoor et al. (2008) que verificaram eficácia da penicilina e cefotaxime no controle desta subespécie isolada de equinos com doença respiratória. No presente estudo 35\% (14/40) dos isolados foram resistentes a cefotaxime, enquanto que $45 \%(18 / 40)$ apresentaram resistência a estreptomicina, $42,5 \%$ (17/40) a imipenem e $40 \%$ (16/40) a ampicilina. Estudos realizados por Morales et al. (2010) em equinos mostraram resistência de $S$. equi subespécie zooepidemicus aos antibióticos amicacina, ampicilina, gentamicina, acido nalidixico e ciprofloxacina, já Kirinus et al. (2011) verificaram suceptibilidade de $100 \%$ dos isolados a ampicilina, imipenem e estreptomicina.

Em relação aos demais antibióticos avaliados (Tabela 3), constatou-se que a resistência foi variável com porcentagens entre 0 a $17,5 \%$, verificando-se significância entre diferentes isolados $(\mathrm{p}<0,05)$.

O surgimento de resistência a diversos antibióticos é de considerável importância médica e pode estar relacionada com a utilização irracional e às vezes sem controle no tratamento de doenças infecciosas (Morales et al., 2010). Por esta razão a utilização dos diferentes antimicrobianos deve ser considerada como uma questão ética na área da saúde, principalmente relacionada a dosagens e administração adequada de cada fármaco (Kirinus et al., 2011). A resistência múltipla aos antimicrobianos pelas bactérias representa um risco potencial para saúde dos animais e uma ameaça a saúde pública.

\section{CONCLUSÕES}

De acordo com a metodologia empregada e pelos resultados obtidos concluiu-se que Streptococcus equi subespécie equi e Streptococcus equi subespécie zooepidemicus estão presentes na cavidade nasal tanto de equinos com doenças respiratórias como também de animais hígidos, podendo ser transmitidas aos seres humanos, o que 
o torna um importante agente zoonótico para a saúde pública.

Também ficou evidente a resistência e multirresistência destes patógenos aos antibióticos testados. A ocorrência de adenite equina e o tratamento instituído a esta doença, que é baseado no uso empírico e indiscriminado de antibióticos, certamente está causando este problema. Por isso, o surgimento de resistência a diversos antibióticos pelos patógenos estudados é de considerável importância médica e representa um risco potencial para saúde dos animais, além de ser uma ameaça à saúde pública.

\section{REFERÊNCIAS}

BAUER, A. W. et al. Antibiotic susceptibility testing by a standardized single disk method. American Journal Clinical Pathology, v. 45, p. 493-496, 1966.

CHRISTLEY, R. M. et al. Acase-control study of respiratory disease in Thoroughbred racehorses in Sidney, Australia. Equine Veterinary Journal, v. 33, n. 3, p. 256-264, 2010.

FACKLAM, R. What happened to the Streptococci: Overview of taxonomic and nomenclature changes. Clinical Microbiology Veterinary, v. 15, p. 613-630, 2002.

FULDE, M.; VALENTIN-WEIGAND, P. Epidemiology and pathogenicity of zoonotic streptococci. Current topics in microbiology and immunology, v. 368, p. 49-81, 2013.

GOEHRING, L. S. et al. Neurological syndromes among horses in The Netherlands. A 5 year retrospective survey (1999-2004). Veterinary Quartery, v. 27, n. 1, p. 11-20, 2005.

HARRINGTON D. J. et al. The molecular basis of Streptococcus equi infection and disease. Microbes and Infection, v. 4, p. 501-510, 2002.

HIRSH, D. C.; ZEE, Y. C. Microbiologia Veterinária, Ed. Guanabara Koogan S.A., 2003. 446p.

HOLDEN M. T. et al. Genomic evidence for the evolution of Streptococcus equi: host restriction, increased virulence, and genetic exchange with human pathogens. PLos Pathology, v. 5, n. 3, p. 1000-1046, 2009.

JANNATABADI, A. A. et al. Molecular identification of Streptococcus equi subsp equi and Streptococcus equi subesp. zooepidemicusin nassal swabs samples from horses suffering respiratory infection in Iran. Pakistan Journal of Biology Science, v. 11, n. 3, p. 468-471, 2008

KONEMAN, E. W. et al. Diagnóstico microbiológico. Guanabara Koogan, Rio de Janeiro, 2001. 1465p.

KIRINUS, J. K. et al. Perfil fenotípico e susceptibilidade antimicrobiana de Streptococcus equi isolados de equinos da região do sul do Brasil. Pesquisa Veterinária Brasileira, v. 31, n. 3 , p. $231-238,2011$

KUWAMOTO, Y. et al. Microplate sugar-fermentation assay distinguishes Streptococcus equi from other streptococci of Lancefield's group C. Journal of Equine Veterinay Science, v. 12 , p. $47-49,2001$.

MANZOOR, S. et al. Occurrence of Lancefield group C streptococcal species in strangles cases of foals in Punjab,
Pakistan. Pakistan Veterinary Journal, v. 8, n. 1, p. 17-20, 2008.

MIR, I. et al. The study of aerobic bacterial flora of de upper respiratory tract of equines from Jammu and Kashmir region of India. Veterinary World, v. 6, p. 623-627, 2013.

MOLONEY, E. et al. Lineages of Streptococcus equi ssp equi in the Irish equine industry. Irish Veterinary Journal, v. 66, n. 10 , p. $1-8,2013$

MORALES, A. B. et al. Múltiple resistencia antibacterial en aislados de equinos pura sangre de carreras en el hipódromo "La Rinconada", Caracas, Venezuela. Revista de Investigación Veterinária del Perú, v. 21, n. 2, p. 187-191, 2010.

NCCLS. Performance Standards for Antimicrobial Disk Susceptibility Tests; Approved Standard- Eighth Edition. NCCLS document M2-A8 [ISBN 1-56238-485-6]. NCCLS, 940 West Valley Road, Suite 1400, Wayne, Pennsylvania 190871898 USA, 2003.)

QUINN, P. J. et al. Veterinary microbiology and microbial disease. 2. Ed: Blackwell Science Ltda, 2011. 512p.

RASMUSSEN, C. D. et al. Streptococcus equi subsp. zooepidemicus isolates from equine infectious endometritis belong to a distinct genetic group. Veterinary Research, v. 44, n. 26 , p. $1-8,2013$.

SCHILD, A. L. Infecção por Streptococcus equi (Garrotilho). In: RIET-CORREA, F. SCHILD, A. L.; MÉNDEZ, M. C.; LEMOS, R. A. A. (Eds.). Doenças de ruminantes e equinos. São Paulo: Varela, 2001. v.1, p. 265-269.

SILVA, F. A. S. E.; AZEVEDO, C. A. V. de. Principal Components Analysis in the Software Assistat-Statistical Attendance. In: WORLD CONGRESS ON COMPUTERS IN AGRICULTURE, 7, Reno-NV-USA: American Society of Agricultural and Biological Engineers, 2009.

SWEENEY, C. R. et al. Streptococcus equi infectios in horses: guidelines for treatment, control, and preventions of strangles. Journal of Veterinary Internal Medicine, v. 123, p. 123-134, 2005.

TIMONEY, J.F. The pathogenic equine streptococci. Veterinary Research, v. 35, p. 397-409, 2004.

TORTORA, G. J.; FUNKE, B. R.; CASE, C. L. Microbiologia. Ed. Artmed, 2012, 934p.

WALLER A. S.; JOLLEY K. A. Getting a grip on strangles: Recent progress towards improved diagnostics and vaccines. Veterinary Journal, v. 173, p. 492-501, 2007.

WALLER, A. S. et al. Streptococcus equi: a pathogen restricted to one host. Journal of Medical Microbiology, v. 60, p. 12311240, 2011.

WEBB, $\mathrm{K}$. et al. Development of an unambiguous and discriminatory multilocus sequence typing scheme for the Streptococcus zooepidemicus group. Microbiology, v. 154, n. 10 , p. 3016-3024, 2008. 